\title{
Breast-Specific $\gamma$-Imaging for the Detection of Mammographically Occult Breast Cancer in Women at Increased Risk
}

\author{
Rachel F. Brem, Rachel C. Ruda, Jialu L. Yang, Caitrín M. Coffey, and Jocelyn A. Rapelyea \\ George Washington University Medical Faculty Associates, Washington, District of Columbia
}

\begin{abstract}
Breast-specific $y$-imaging (BSGI) is a physiologic imaging modality that can detect subcentimeter and mammographically occult breast cancer, with a sensitivity and specificity comparable to MRI. The purpose of this study was to determine the incremental increase in breast cancer detection when BSGI is used as an adjunct to mammography in women at increased risk for breast cancer. Methods: All patients undergoing BSGI from April 2010 through January 2014 were retrospectively reviewed. Eligible patients were identified as women at increased risk for breast cancer and whose most recent mammogram was benign. Examinations exhibiting focally increased radiotracer uptake were considered positive. Incremental increase in cancer detection was calculated as the percentage of mammographically occult BSGI-detected breast cancer and the number of mammographically occult breast cancers detected per 1,000 women screened. Results: Included in this study were 849 patients in whom 14 BSGl examinations detected mammographically occult breast cancer. Patients ranged in age from 26 to $83 \mathrm{y}$, with a mean age of $57 \mathrm{y}$. Eleven of 14 cancers were detected in women with dense breasts. The addition of BSGI to the annual breast screen of asymptomatic women at increased risk for breast cancer yields 16.5 cancers per 1,000 women screened. When high-risk lesions and cancers were combined, BSGI detected 33.0 high-risk lesions and cancers per 1,000 women screened. Conclusion: BSGI is a reliable adjunct modality to screening mammography that increases breast cancer detection by $1.7 \%(14 / 849)$ in women at increased risk for breast cancer, comparable to results reported for breast MRI. BSGI is beneficial in breast cancer detection in women at increased risk, particularly in those with dense breasts.
\end{abstract}

Key Words: breast-specific gamma imaging; breast cancer; scintimammography; molecular breast imaging

J Nucl Med 2016; 57:678-684

DOI: 10.2967/jnumed.115.168385

B reast cancer is the second most common cancer in American women (1). X-ray mammography remains the standard of breast cancer screening and is an effective imaging tool that reduces mortality from breast cancer (2). However, it is an imperfect tool,

Received Oct. 19, 2015; revision accepted Dec. 10, 2015.

For correspondence or reprints contact: Rachel F. Brem, George Washington University, Medical Faculty Associates, 2300 M St. NW, 8th FI., Washington, DC 20037.

E-mail: rbrem@mfa.gwu.edu

Published online Jan. 28, 2016.

COPYRIGHT (C 2016 by the Society of Nuclear Medicine and Molecular Imaging, Inc. with an overall reported sensitivity of $85 \%$, which decreases to $68 \%$ in women with dense breasts $(3,4)$. In prospective trials among women at high risk for breast cancer due to a familial or genetic predisposition, mammography demonstrated a $30 \%-$ $40 \%$ sensitivity (5). Because of the limitations of mammography, supplemental imaging modalities, including MRI, whole-breast screening ultrasound, and breast-specific $\gamma$-imaging (BSGI), are becoming increasingly important for women at increased risk, with the goal of detecting early-stage breast cancer.

Breast MRI is a physiologic imaging modality recommended as a supplemental screening tool to mammography for high-risk women ( $\geq 20 \%-25 \%$ lifetime risk) (6). MRI has demonstrated an incremental detection rate of 9.5 cancers per 1,000 high-risk women screened (7) and variable sensitivity and specificity ranging from $71 \%$ to $92 \%$ and $54 \%$ to $86 \%$, respectively $(8-10)$. However, MRI is costly, time-consuming for radiologists to interpret, poorly tolerated by some patients due to claustrophobia, and inaccessible to patients who are obese, have implanted devices, or have renal insufficiency. Whole-breast ultrasound has also been studied as a supplemental screening tool, improving sensitivity from $50 \%$ to $77.5 \%$ when used with mammography (11). It has been shown to yield an additional 1.9-3.25 detected cancers per 1,000 women screened, compared with screening mammography alone (12-14). However, the use of whole-breast ultrasound is correlated with a higher callback and false-positive biopsy rate (15).

Like MRI, BSGI is a physiologic imaging tool used to detect breast cancer. BSGI has demonstrated a sensitivity of $92 \%-96 \%$ and a specificity of $71 \%-80 \%(8,16,17)$ and has been shown to reliably detect mammographically occult breast cancers (18). BSGI uses a radiotracer, ${ }^{99 \mathrm{~m}} \mathrm{Tc}$-sestamibi, to identify physiologic differences between malignant and normal breast tissue. ${ }^{99 \mathrm{~m}} \mathrm{Tc}-$ sestamibi $\gamma$-imaging, when used with mammography for breast cancer screening in women at increased risk and with dense breasts, significantly improves the sensitivity and positive predictive value as well as increases the number of breast cancers detected by $7.5-8.8$ per 1,000 women $(19,20)$. Furthermore, BSGI has been shown to detect additional foci of mammographically occult breast cancer in $9 \%$ of women with newly diagnosed breast cancer (21). A comprehensive meta-analysis of relevant studies from 1984 to 2012 concluded that BSGI detected mammographically occult cancer in $4 \%$ of patients with benign mammograms and additional cancers in $6 \%$ of those with abnormal mammograms or new biopsy-proven breast cancer (17). A preliminary review of a population of high-risk women with benign mammographic imaging $(n=94)$ found that this modality was able to 
detect small $(<1 \mathrm{~cm})$, mammographically occult lesions in women at increased risk for breast cancer (22).

To date, no large-scale studies have been published examining the clinical utility of BSGI in women at increased risk for breast cancer. The purpose of this study was to determine the incremental increase in breast cancer detection when using BSGI in addition to mammography for women at increased risk of developing breast cancer.

\section{MATERIALS AND METHODS}

\section{Study Population}

This study was approved by the Institutional Review Board and is Health Insurance Portability and Accountability Act-compliant. All results and data were obtained retrospectively from patients' medical records with waived patient consent. All women who had a BSGI examination from April 2010 through January 2014 were retrospectively reviewed. Patients were included who were determined to be at increased risk for breast cancer and whose most recent screening or diagnostic mammogram was negative or probably benign (BI-RADS 1,2 , or 3 ). Women were identified as being at increased risk if they had one or more of the following risk factors: a personal history of breast cancer, a known mutation in the BRCA1 or BRCA2 gene, a family history of breast cancer in at least 1 first-degree or 2 seconddegree relatives, a history of axillary or mediastinal irradiation, or a personal history of an atypical high-risk lesion, including atypical ductal hyperplasia $(\mathrm{ADH})$, atypical lobular hyperplasia, lobular carcinoma in situ, papilloma, flat epithelial atypia, or radial scar.

Patients were excluded who were symptomatic; had newly diagnosed breast cancer; had breast surgery or biopsy up to 1 mo before the BSGI, because the effects of postbiopsy or postsurgical changes have not yet been established; or whose most recent mammogram was abnormal. Patients whose most recent mammogram was abnormal or incomplete, but whose follow-up with ultrasound, MRI, or biopsy was negative or benign were included because there was documentation that the mammographic abnormality was not due to cancer, provided that the biopsy was 3 or more months before the BSGI. For patients who routinely received MRI for high-risk screening, their most recent mammogram or MRI must have been negative or probably benign to qualify for inclusion. Patients undergoing treatment for breast cancer at the time of their BSGI were not included, although patients on chemopreventatives such as tamoxifen were included.

\section{Breast Density}

Breast density was determined by the patient's most recent mammography report before BSGI. Breast density reported as fatty replaced or scattered fibroglandular tissue (BI-RADS a or b) was classified as nondense, and density reported as heterogeneously dense or extremely dense (BI-RADS c or d) was classified as dense (23).

\section{BSGI}

All BSGI examinations were performed with a single-head, highresolution breast-specific $\gamma$-camera (6800; Dilon Technologies). Patients were imaged in a seated position. Patients were injected in the dorsal venous complex of the hand or antecubital vein with radiotracer, and imaging began immediately after injection of the radiotracer. Initially, a mean of $781 \mathrm{MBq}(21.1 \mathrm{mCi})$ (high dose; range, 592-1,188 MBq [16.0-32.1 mCi]) of 99m Tc-sestamibi (Miraluma; Radiology Services of Northern Virginia) was used $(n=653)$. However, in November 2012 the protocol was modified to use a mean of 296 $\mathrm{MBq}(8.0 \mathrm{mCi})$ (low-dose; range, 259-500 MBq [7.0-13.5 mCi]) of radiotracer $(n=196)$. Images were obtained in the craniocaudal and mediolateral oblique views for a minimum of 100,000 counts per image, with imaging beginning immediately after injection of the radiotracer isotope. Average acquisition time for each image ranged from 6 to $10 \mathrm{~min}$ for a total imaging time of approximately $40 \mathrm{~min}$ per study.

The initial craniocaudal and mediolateral oblique images were reviewed, and additional views were obtained as deemed necessary by the interpreting radiologist. No additional radiotracer injection was used for additional imaging.

\section{Interpretation of BSGI}

All BSGI examinations were reviewed by 3 board-certified radiologists with a range of 6-20 y of experience in BSGI interpretation. Images were interpreted with access to patient history and adjunct imaging studies. Examinations were not reinterpreted for the purposes of this study. BSGI examinations with no focally increased radiotracer uptake, scattered heterogeneous uptake, or stable uptake compared with previous benign BSGI were classified as normal, whereas those with an area of focally increased radiotracer uptake were classified as abnormal. Images were assigned a score of $0-5$, paralleling the BIRADs assessment categories at the time of interpretation (23). Scores of 1,2 , or 3 were classified as a negative BSGI examination, and scores of 0,4 , or 5 , were classified as positive for purposes of analysis (Fig. 1) (24).

\section{Data Collection and Analysis}

Risk factors for breast cancer; age; race; menopausal status; breast density as noted in the patient's most recent mammogram report; BSGI results, including specific location of any areas of abnormal radiotracer uptake; and final pathology from either the minimally invasive biopsy or the surgical excision were recorded for each patient. The data were analyzed to determine the number of asymptomatic women with mammographically occult BSGI-detected breast cancer. Incremental

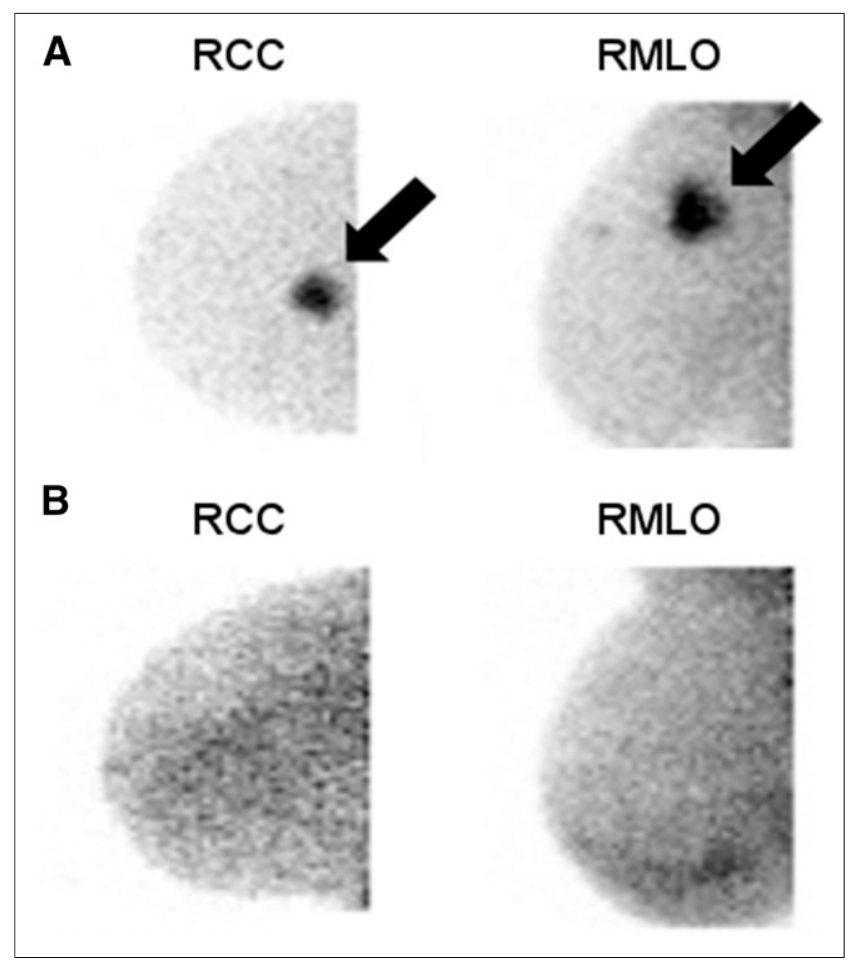

FIGURE 1. Positive versus negative BSGl imaging. (A) Positive BSGI in right craniocaudal (RCC) and mediolateral oblique (RMLO) views exhibiting area of focally increased radiotracer uptake. (B) Negative BSGI in RCC and RMLO views exhibiting no areas of focally increased radiotracer uptake. 
TABLE 1

Population of Women at Increased Risk for Breast Cancer

\begin{tabular}{lccc}
\hline \multicolumn{1}{c}{ Risk factor } & $\begin{array}{c}\text { Increased-risk } \\
\text { population }(n=849)\end{array}$ & BSGI-positive population $(n=212)$ & Malignant population $(n=14)$ \\
\hline Family history & $230(27.1 \%)$ & $67(31.6 \%)$ & $7(50.0 \%)$ \\
\hline Personal history & $430(50.7 \%)$ & $89(41.98 \%)$ & $1(7.1 \%)$ \\
Atypia & $54(6.4 \%)$ & $20(9.4 \%)$ & $2(14.3 \%)$ \\
\hline BRCA1/2 & $2(0.2 \%)$ & $0(0.0 \%)$ & $0(0.0 \%)$ \\
\hline Family + personal history & $83(9.8 \%)$ & $19(9.0 \%)$ & $1(7.1 \%)$ \\
\hline Family history + atypia & $28(3.3 \%)$ & $13(6.1 \%)$ & $2(14.3 \%)$ \\
\hline Personal history + atypia & $10(1.2 \%)$ & $2(0.9 \%)$ & $0(0.0 \%)$ \\
\hline Family history + BRCA1/2 & $6(0.7 \%)$ & $1(0.5 \%)$ & $0(0.0 \%)$ \\
\hline Personal history + BRCA1/2 & $2(0.2 \%)$ & $1(0.5 \%)$ & $1(7.1 \%)$ \\
\hline 3 or more risk factors & $4(0.5 \%)$ & $0(0.0 \%)$ & $0(0.0 \%)$ \\
\hline
\end{tabular}

increase in breast cancer detection was calculated using Microsoft Excel as the number of women who had breast cancer detected on BSGI of all women studied. PPV 1 was calculated as the number of malignancies per all abnormal BSGI examinations, and $\mathrm{PPV}_{3}$ was calculated as the number of malignancies per all biopsies performed. $\left(\mathrm{PPV}_{1}\right.$ is the positive predictive value for abnormal findings [number of malignant results out of total number of positive BSGI exams] and $\mathrm{PPV}_{2}$ is the positive predictive value for total biopsies performed [number of malignant results out of total number of biopsies].) The statistical significance of the difference in cancer detection rate between both high-dose and low-dose and dense and nondense subgroups of the population was performed using $\chi^{2}$ tests calculated by SPSS Statistics (IBM). All $P$ values were reported as 2 -sided. A $P$ value of less than 0.05 was set as the threshold value for a significant difference. The data were further analyzed with Microsoft Excel for positive predictive values. For the reference standard, either pathologic results of biopsy or follow-up imaging within $1 \mathrm{y}$ that did not demonstrate evidence of malignancy were used.

\section{RESULTS}

A total of 1,723 women had BSGI examinations between April 2010 and January 2014. Of these, 849 patients (49.3\%) were at increased risk with benign prior mammograms and were included in this study. Patients ranged in age from 26 to $83 \mathrm{y}$, with a mean age of $57 \mathrm{y}$.

\section{Risk Factors}

Among the 849 patients at increased risk who had BSGI examinations, $230(27.0 \%)$ had a family history of breast cancer and $430(50.6 \%)$ had a personal history of breast cancer. Fifty-four $(6.3 \%)$ had a personal history of an atypical, high-risk lesion. Two of 849 patients had a known mutation in BRCA 1 or $2(0.2 \%)$; 1 was BRCA1-positive, and 1 was BRCA2-positive. There were 133 of 849 patients $(15.6 \%)$ with 2 or more of these risk factors and 4 of $849(0.5 \%)$ patients with 3 of these risk factors.

Of the 849 patients at increased risk, $212(25.0 \%)$ had positive BSGI examinations, demonstrating focally increased radiotracer uptake. Of the patients with BSGI-positive results, 67 (31.6\%) had a family history of breast cancer and $89(42.0 \%)$ had a personal history of breast cancer and $20(9.4 \%)$ had a personal history of an atypical, high-risk lesion. Neither of the patients with BRCA gene mutations had abnormal BSGI examinations. Thirty-six of 212 patients $(17.0 \%)$ had 2 or more of these risk factors, and no patients with BSGI-positive examinations had 3 risk factors.

Fourteen BSGI-positive patients were found to have mammographically occult, BSGI-detected breast cancer. One of 14 (7.14\%) had a family history of breast cancer, 7 of $14(50.0 \%)$ had a personal history of breast cancer, and 2 of $14(14.3 \%)$ had a history of atypia. Four of 14 patients $(28.6 \%)$ had 2 or more of these risk factors (Table 1).

\section{Additional Imaging}

Of the 849 patients at increased risk who had BSGI examinations, $637(75.0 \%)$ were negative, stable, or likely benign (scores of 1,2 , or 3 ), with no focally increased uptake, scattered heterogeneous uptake, or stable uptake.

TABLE 2

Biopsy Results $\left(n=97^{\star}\right)$

\begin{tabular}{|c|c|}
\hline Result & $n$ \\
\hline \multicolumn{2}{|c|}{ Benign pathology $(n=66)$} \\
\hline Benign breast tissue & 8 \\
\hline Cyst contents & 7 \\
\hline Fibrocystic changes & 45 \\
\hline Fibroadenoma & 6 \\
\hline \multicolumn{2}{|c|}{ Benign high-risk pathology $(n=14)$} \\
\hline $\mathrm{ADH}$ & 3 \\
\hline Lobular neoplasia & 5 \\
\hline Flat epithelial atypia & 1 \\
\hline Papilloma & 4 \\
\hline Radial scar & 1 \\
\hline \multicolumn{2}{|c|}{ Malignant pathology $(n=14)$} \\
\hline DCIS & 8 \\
\hline IDC & 5 \\
\hline ILC & 1 \\
\hline
\end{tabular}

*3 patients had samples insufficient for diagnosis.

IDC = invasive ductal carcinoma; ILC = invasive lobular carcinoma. 


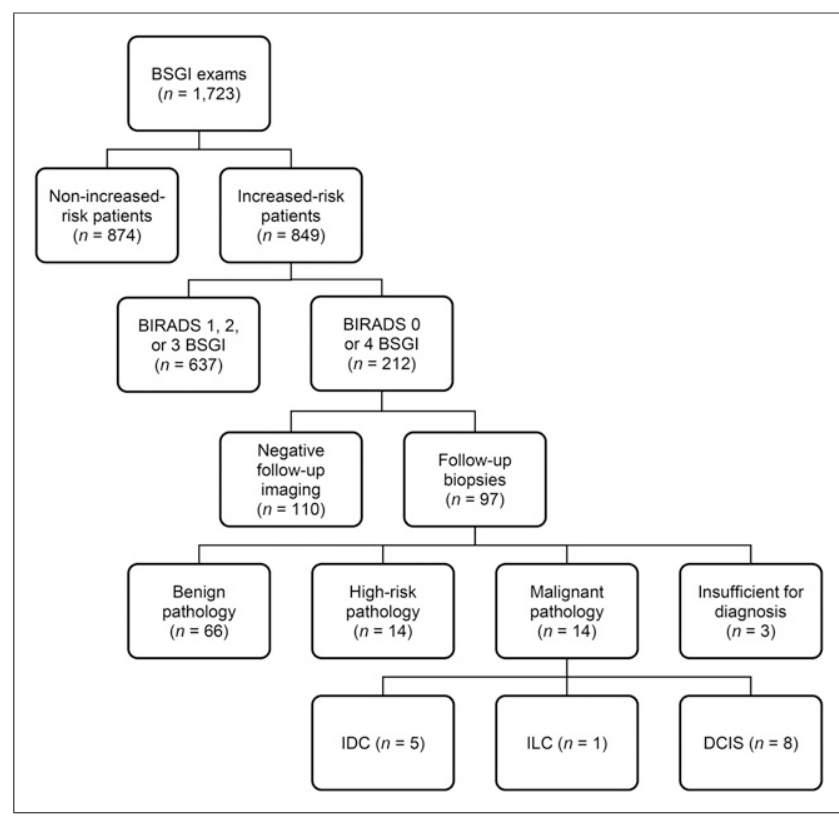

FIGURE 2. BSGI examination outcomes.

Two hundred twelve of the 849 BSGI examinations were positive (scores of 0 or 4 ). Among those with positive examinations, 110 of $212(51.9 \%)$ positive examinations were found to be benign through additional imaging: directed ultrasound was negative in 83 patients; directed second-look mammography was negative in 10 patients, demonstrating either stable fibroadenoma, stable intramammary lymph nodes, or stable benign calcifications; breast MRI was negative in 16 patients; and repeated BSGI at a different time in the patient's menstrual cycle was negative in 1 patient. Additionally, 1 woman with a personal history of breast cancer in the contralateral breast underwent prophylactic mastectomy, which demonstrated benign fibrocystic findings. All patients with negative additional imaging were confirmed benign by mammographic, sonographic, or MR imaging follow-up (1 y after abnormal BSGI).

Additional imaging was abnormal in 99 of 212 (46.7\%) of the patients with BSGI-positive examinations and was unavailable for
2 patients with positive BSGI examinations (1.4\%). Ninetyseven of the 212 BSGI-positive patients (45.7\%) underwent biopsies: ultrasound-guided biopsy in 43 , fine-needle aspiration in 19, stereotactic biopsy in 7 subsequent to directed secondlook mammography, BSGI-guided biopsy in 10 (BSGI-guided biopsy has been offered in our imaging facility since May 2011), MRI-guided biopsy in 16, and surgical excisional biopsy in 2 patients. Two BSGI-positive patients with abnormal additional imaging did not undergo biopsy at our facility, and their pathologic reports were not available.

\section{Benign Biopsy Results}

Biopsy due to BSGI findings was benign in 66 of 97 patients (68.0\%). Benign findings included fibrocystic changes $(n=45)$, benign breast tissue $(n=8)$, fibroadenoma $(n=6)$, and cyst contents $(n=7)$. Three of the patients who underwent biopsy with fine-needle aspiration resulted in samples that were insufficient for diagnosis; 2 of these patients received follow-up imaging revealing benign findings, ruling out the need for repeated biopsy, and 1 patient underwent a follow-up BSGI-guided biopsy yielding benign pathology.

Benign high-risk lesions were found in 14 of 97 (14.4\%) of biopsied lesions: $\operatorname{ADH}(n=3)$, atypical lobular hyperplasia $(n=2)$, lobular carcinoma in situ $(n=3)$, radial scar $(n=1)$, flat epithelial atypia $(n=1)$, and papillary lesion $(n=4)$. All high-risk lesions were subsequently surgically excised. This study found that BSGI detects 16.5 high-risk lesions for every 1,000 patients at increased risk screened for breast cancer.

\section{Malignant Biopsy Results}

Of 849 patients at increased risk, BSGI detected $14(1.7 \%)$ mammographically occult breast cancers, with a $\mathrm{PPV}_{1}$ of $6.7 \%$ (14/212) and $\mathrm{a} \mathrm{PPV}_{3}$ of $14.4 \%$ (14/97). Eleven of the 14 cancers (11.3\%) were diagnosed at initial biopsy; 3 high-risk lesions (1 radial scar, $2 \mathrm{ADH})$ upgraded at surgical excision to ductal carcinoma in situ (DCIS) (Table 2). The other cancers detected were invasive ductal carcinoma $(n=5)$, invasive lobular carcinoma $(n=1)$, and DCIS $(n=8)$ (Fig. 2). Invasive breast cancers ranged in size from 0.3 to $4.0 \mathrm{~cm}$ (Table 3). Of the 14 BSGI-detected cancers, biopsy was performed under ultrasound guidance in 8 , MRI guidance in 2, BSGI guidance in 2, and surgical excision in 2. This study found

TABLE 3

Characteristics of Invasive Cancers $(n=6)$

\begin{tabular}{cccccc}
\hline Type & Grade & Size $(\mathrm{cm})$ & Hormones & Density & Risk factors \\
\hline IDC & 2 & 2.0 & ER/PR/Her2- & 4 & PH radial scar \\
IDC & 2 & 2.5 & ER/PR+, Her2- & 3 & PH cancer \\
IDC & 1 & Unavailable* & Unavailable* & 3 & FH cancer, PH ADH, PH LCIS \\
IDC & 3 & 0.7 & ER/PR-, Her2+ & 3 & PH cancer \\
IDC & 3 & 0.3 & ER/PR/Her2- & 3 & PH cancer, BRCA2+ \\
ILC & 3 & 4.0 & ER/PR,+ Her2- & 3 & FH cancer
\end{tabular}

*Ultrasound-guided core-needle breast biopsy was performed. Subsequent surgical excision was performed at outside facility. Final pathologic report from surgery is not available.

$\mathrm{IDC}=$ invasive ductal carcinoma; $\mathrm{ER}=$ estrogen receptor; $\mathrm{PR}=$ progesterone receptor; Her2 = human epidermal growth factor receptor 2; $\mathrm{PH}=$ personal history; $\mathrm{FH}$ = family history; LCIS = lobular carcinoma in situ; ILC = invasive lobular carcinoma. 
that BSGI detects 16.5 mammographically occult cancers for every 1,000 patients at increased risk screened for breast cancer.

When high-risk lesions and cancers were combined, BSGI detected an additional 33.0 cancers and high-risk lesions for every 1,000 patients screened at increased risk for breast cancer.

\section{High-Dose Versus Low-Dose BSGI Injections}

When results of patients who received low-dose versus highdose BSGI injections were compared, low-dose examinations yielded 2 cancers of 196 examinations, with a $P P V_{1}$ and $P_{P V}$ of $3.9 \%(2 / 51)$ and $10.5 \%(2 / 19)$, respectively, whereas high-dose examinations yielded 12 cancers of 653 examinations, with a $\mathrm{PPV}_{1}$ and $\mathrm{PPV}_{3}$ of $7.4 \%$ (12/161) and $15.4 \%$ (12/78), respectively. BSGI detected 18.4 and 10.2 mammographically occult cancers for every 1,000 patients screened among patients receiving high and low doses of injections, respectively. Analysis of the difference in cancer detection rate between low-dose and high-dose BSGI examinations yielded an $\chi^{2}$ value of 0.62 , which was not statistically significant (95\% confidence interval, $0.12-2.48$; $P=0.44)$.

\section{Breast Tissue Density}

Of the 849 patients at increased risk, 302 patients $(35.6 \%)$ had nondense breast tissue; 64 patients had fatty replaced breast tissue (BI-RADS a) and 238 had scattered fibroglandular breast tissue (BI-RADS b). Three of 14 breast cancers were detected in women with nondense breast tissue $(21.4 \%)$. Among women with nondense breasts, BSGI detected a mammographically occult breast cancer in 3 of 302 patients (1.0\%), or 9.9 cancers per 1,000 women screened.

Five hundred forty-seven patients $(64.4 \%)$ had dense breast tissue, 445 patients with heterogeneously dense breast tissue (BIRADS c) and 102 patients with extremely dense tissue (BI-RADS d). Eleven of 14 breast cancers detected by BSGI in the study occurred in women with dense breast tissue $(78.6 \%)$. Among women with dense breasts, BSGI detected a mammographically occult breast cancer in 11 of 547 patients $(2.0 \%)$, or 20.1 cancers per 1,000 women screened (Table 4). Analysis of the difference in cancer detection rate in women with dense and nondense breast tissue yielded a $\chi^{2}$ value of 1.24 (95\% confidence interval, 0.14 $1.77 ; P=0.28$ ), which was not statistically significant. This is similar to data previously published that BSGI demonstrates no difference in detecting cancers in women with dense and nondense breasts (25).

\section{DISCUSSION}

In this retrospective review of 849 patients at increased risk for breast cancer with benign mammograms, 212 (25.0\%) had positive BSGI examinations. This recall rate falls within the range of previously reported BSGI recall rates in women with benign mammograms $(22,26)$. BSGI detected an additional 14 mammographically occult breast cancers $(1.7 \%)$, or 16.5 cancers per 1,000 women screened. This is comparable to prior reports for MRI detection of occult cancer in high-risk populations of 9.5 cancers per 1,000 women screened (7). Among women with personal histories of atypia including lobular carcinoma in situ and $\mathrm{ADH}$, incremental detection rates using MRI range from $1.6 \%$ to $4.5 \%$ (27-29).

Our findings suggest that the addition of BSGI can improve the ability to detect breast cancer in women at increased risk compared with mammography alone. Eleven of 14 (78.6\%) mammographically occult cancers were detected in patients with heterogeneous or extremely dense breast tissue (BI-RADS $\mathrm{c}$ or d). This supports existing data, which has demonstrated that unlike mammography, the detection of breast cancer with BSGI is not affected by breast density (25).

Among the 14 mammographically occult, BSGI-detected cancers found in this study, 8 were DCIS $(57.1 \%)$ and 6 were invasive carcinomas (42.9\%) ranging in size from 0.3 to $4.0 \mathrm{~cm}$ (Table 3). Because of the high sensitivity of BSGI in detecting both invasive and noninvasive cancers, BSGI is a valuable supplemental imaging tool to mammography in detecting breast cancer in women at increased risk.

\section{BI-RADS 3 Interval Imaging}

Patients were included in this study whose most recent mammogram was negative or probably benign (BI-RADS 1, 2, or 3). Thirty patients with BSGI-positive examinations received BI-RADS 3 diagnoses on their mammogram reports. Patients with BI-RADS 3 scores were indicated for 6-mo-interval screening for reasons including postsurgical changes, asymmetry, and calcifications. Of these 30 patients, 3 were subsequently diagnosed with atypia, and 3 were diagnosed with cancer. Because patients with BIRADS 3 mammogram scores are recommended for various interval screening options (mammogram, ultrasound, MRI, BSGI) depending on their histories and diagnoses, these data suggest that BSGI is a viable interval screening option for women at increased risk for the earlier detection of breast cancer.

\section{BSGI Versus MRI}

Surveillance of patients at a higher risk for the development of breast cancer can also be achieved via screening MRI as an adjunct modality to mammography. However, MRI is more expensive, requires more interpretation time, is poorly tolerated by some patients because of severe claustrophobia, and is inaccessible to patients with renal insufficiency, implanted devices, or large body habitus. Nevertheless, MRI has advantages, including the absence of radiation and greater availability.

Studies have demonstrated that BSGI and MRI have similar sensitivity and specificity in breast cancer detection. MRI has been shown to have a sensitivity of $71 \%-99 \%$ and a specificity of $54 \%-95 \%(8-10)$. BSGI detects breast cancer with a sensitivity of $92 \%-96 \%$ and a specificity of $71 \%-80 \%(7,16,17)$. In a study comparing DCIS detection using BSGI, MRI, and mammography, sensitivity for detecting DCIS was found to be slightly

TABLE 4

Breast Density in Patients Diagnosed with Cancer

\begin{tabular}{lcc}
\hline Breast tissue & No. of patients & No. of patients with mammographically occult, BSGI-detected breast cancer \\
\hline Nondense & 302 & $3 / 302(1.0 \%)$ \\
Dense & 547 & $11 / 547(2.0 \%)$ \\
\hline
\end{tabular}


higher with BSGI (91\%) than with MRI (88\%) or mammography $(82 \%)(30,31)$.

Studies have shown a similar incremental increase in breast cancer detection among high-risk women with MRI and BSGI: 9.5 cancers per 1,000 women screened with MRI and 7.5-8.8 cancers per 1,000 women with BSGI $(7,19,20)$. Our study demonstrates that BSGI detects occult breast cancer in women at increased risk at a rate of 16.5 cancers detected per 1,000 women screened, higher than the rate reported for both MRI and screening ultrasound in women at increased risk for breast cancer (11). However, this difference may be due to a smaller sample size in our study. Additional larger studies are needed to further assess the incremental cancer detection rate with BSGI in women at increased risk.

\section{Risk from Radiation}

The primary disadvantage of BSGI is the radiation exposure. However, when used as an adjunct in increased-risk populations, this is a reasonable imaging option. One study showed that a dose of $300 \mathrm{MBq}$ or $8.1 \mathrm{mCi}$ was sufficient in the detection of breast cancer using BSGI, and further studies are being conducted to reduce the dose of radiotracer needed without compromising image quality $(20,32)$. In this study population, a dose of 592$1,188 \mathrm{MBq}(16.0-32.1 \mathrm{mCi})$ of ${ }^{99 m} \mathrm{Tc}$-sestamibi was originally used, which was reduced to $259-500 \mathrm{MBq}(7.0-13.5 \mathrm{mCi})$ of ${ }^{99 \mathrm{~m}} \mathrm{Tc}$ sestamibi part way through the study. The differences in positive predictive value and incremental cancer detection between high and low doses were not statistically significant. In women who cannot or will not undergo MRI, the availability of BSGI gives women the option of physiologic imaging.

\section{Treatment and Outcome of Atypical, High-Risk Lesions}

Of the patients in this study with positive BSGI examinations, 35 of $212(16.5 \%)$ had personal histories of atypical, high-risk lesions. Of the patients diagnosed with cancer, 4 of $14(28.6 \%)$ had a history of atypia. In this study, the upgrade rate from atypical lesions to cancer due to a finding on BSGI was $21.4 \%(3 / 14)$. This is comparable to existing data demonstrating upgrade rates of $20 \%-23 \%$ for MRI-detected atypia $(33,34)$. These findings suggest that surgical excision should be recommended for all atypical, highrisk lesions found on BSGI. Additional studies are needed to determine the optimal management of BSGI-detected high-risk lesions.

Limitations of this study include an absence of long-term follow-up to determine false-negative BSGI studies or subsequent malignant findings in the 2 instances in which the patient declined a biopsy. Additionally, this is a retrospective study, and the time intervals between mammograms and BSGI examinations are varied. Furthermore, the change in radiotracer dose during the study period may have affected findings. However, the difference in incremental increase in cancer detection between the high- and low-dose groups was not statistically significant.

\section{CONCLUSION}

This study demonstrates that BSGI detects mammographically occult breast cancer in $1.7 \%$ of women at increased risk of breast cancer with negative mammograms, or 16.5 additional cancers per 1,000 women screened in this population. It further finds that the use of BSGI detects a combined 33.0 high-risk lesions and cancers per 1,000 women screened. The detection of mammographically occult breast cancer was greater in women with dense breasts, but BSGI also detected additional cancers in women with nondense breast tissue. Furthermore, this study suggests that high-risk women with BI-RADS 3 mammograms may benefit from BSGI in the earlier detection of breast cancer.

This study supports the use of BSGI as a supplemental modality to mammography in women at increased risk for breast cancer, particularly for those with dense breast tissue or in whom MRI cannot be performed.

\section{DISCLOSURE}

The costs of publication of this article were defrayed in part by the payment of page charges. Therefore, and solely to indicate this fact, this article is hereby marked "advertisement" in accordance with 18 USC section 1734. Rachel Brem, is on the Board of Managers of and has stock in Dilon Technologies. This research was approved by the GWU Institutional Review Board. No other potential conflict of interest relevant to this article was reported.

\section{REFERENCES}

1. American Cancer Society. Breast Cancer Facts and Figures: 2011-2012. American Cancer Society website. http://www.cancer.org/acs/groups/content/@epidemiology surveilance/documents/document/acspc-030975.pdf. Accessed March 23, 2016.

2. Humphrey LL, Helfand M, Chan BK, Woolf SH. Breast cancer screening: a summary of the evidence for the US Preventive Services Task Force. Ann Intern Med. 2002;137:347-360.

3. Rosenberg RD, Hunt W, et al. Effects of age, breast density, ethnicity, and estrogen replacement therapy on screening mammographic sensitivity and cancer stage at diagnosis: review of 183,134 screening mammograms in Albuquerque, New Mexico. Radiology. 1998;209:511-518.

4. Kolb TM, Lichy J, Newhouse JH. Comparison of the performance of screening mammography, physical examination, and breast US and evaluation of factors that influence them: an analysis of 27,825 patient evaluations. Radiology. 2002;225:165-175.

5. Berg WA, Zhang Z, Lehrer D, et al. Detection of breast cancer with addition of annual screening ultrasound or a single screening MRI to mammography in women with elevated breast cancer risk. JAMA. 2012;307:1394-1404.

6. Saslow D, Boetes C, Burke W, et al. American Cancer Society guidelines for breast screening with MRI as an adjunct to mammography. CA Cancer J Clin. 2007;57:75-89.

7. Kriege M, Brekelmans CT, Boetes C, et al. Magnetic resonance imaging screening study group: efficacy of MRI and mammography for breast-cancer screening in women with a familial or genetic predisposition. $N$ Engl J Med. 2004;351:427-437.

8. Johnson N, Sorenson L, Bennetts L, et al. Breast-specific gamma imaging is a cost effective and efficacious imaging modality when compared with MRI. Am J Surg. 2014;207:698-701.

9. Raikhlin A, Curpen B, Warner E, Betel C, Wright B, Jong R. Breast MRI as an adjunct to mammography for breast cancer screening in high-risk patients: retrospective review. AJR. 2015;204:889-897.

10. Weinstein SP, Localio AR, Conant EF, et al. Multimodality screening of highrisk women: a prospective cohort study. J Clin Oncol. 2009;27:6124-6128.

11. Berg WA, Blume JD, Cormack JB, et al. Combined screening with ultrasound and mammography vs mammography alone in women at elevated risk of breast cancer. JAMA. 2008;299:2151-2163.

12. Brem RF, Tabár L, Duffy SW, et al. Assessing improvement in detection of breast cancer with three-dimensional automated breast US in women with dense breast tissue: the SomoInsight Study. Radiology. 2015;274:663-673.

13. Hooley RJ, Greenberg KL, Stackhouse RM, Geisel JL, Butler RS, Philpotts LE. Screening US in patients with mammographically dense breasts: initial experience with Connecticut Public Act 09-41. Radiology. 2012;265:59-69.

14. Weigert J, Steenbergen S. The Connecticut experiment: the role of ultrasound in the screening of women with dense breasts. Breast J. 2012;18:517-522.

15. Brem RF, Lenihan MJ, Lieberman J, Torrente J. Screening breast ultrasound: past, present, and future. AJR. 2015;204:234-240.

16. Brem RF, Petrovitch I, Rapelyea JA, et al. Breast-specific gamma imaging with $99 \mathrm{~m}$-Tc-sestamibi and magnetic resonance imaging in the diagnosis of breast cancer: a comparative study. Breast J. 2007;13:465-469. 
17. Sun Y, Wei W, Yang HW, Liu JL. Clinical usefulness of breast-specific gamma imaging as an adjunct modality to mammography for diagnosis of breast cancer: a systemic review and meta-analysis. Eur J Nucl Med Mol Imaging. 2013;40:450-463.

18. Zhou M, Johnson N, Gruner S, et al. Clinical utility of breast-specific gamma imaging for evaluating disease extent in the newly diagnosed breast cancer patient. Am J Surg. 2009;197:159-163.

19. Rhodes DJ, Hruska CB, Phillips SW, et al. Dedicated dual-head gamma imaging for breast cancer screening in women with mammographically dense breasts. Radiology. 2011;258:106-118.

20. Rhodes DJ, Hruska CB, Conners AL, et al. Journal club: molecular breast imaging at reduced radiation dose for supplemental screening in mammographically dense breasts. AJR. 2015;204:241-251.

21. Brem RF, Shahan C, Rapleyea JA, et al. Detection of occult foci of breast cancer using breast-specific gamma imaging in women with one mammographic or clinically suspicious breast lesion. Acad Radiol. 2010;17:735-743.

22. Brem RF, Rapelyea JA, Zisman G, et al. Occult breast cancer: scintimammography with high-resolution breast-specific gamma camera in women at high-risk for breast cancer. Radiology. 2005;237:274-280.

23. Sickles EA, D’Orsi CJ, Bassett LW, et al. ACR BI-RADS ${ }^{\circledR}$ mammography. In: ACR BI-RADS® Atlas, Breast Imaging Reporting and Data System. Reston, VA: American College of Radiology; 2013:121-140.

24. Conners AL, Hruska CB, Tortorelli CL, et al. Lexicon for standardized interpretation of gamma camera molecular breast imaging: observer agreement and diagnostic accuracy. Eur J Nucl Med Mol Imaging. 2012;39:971-982.

25. Rechtman LR, Lenihan MJ, Lieberman JH, et al. Breast-specific gamma imaging for the detection of breast cancer in dense versus nondense breasts. AJR. 2014;202:293-298.
26. Weigert JM, Bertrand ML, Lanzkowsky L, Stern LH, Kieper DA. Results of a multicenter patient registry to determine the clinical impact of breast-specific gamma imaging, a molecular breast imaging technique. AJR. 2012;198:W69-W75.

27. Morris EA, Liberman L, Ballon DJ, et al. MRI of occult breast carcinoma in a high-risk population. AJR. 2003;181:619-626.

28. Port ER, Park A, Borgen PI, Morris E, Montgomery LL. Results of MRI screening for breast cancer in high-risk patients with LCIS and atypical hyperplasia. Ann Surg Oncol. 2007;14:1051-1057.

29. Sung JS, Malak SF, Bajaj P, Alis R, Dershaw DD, Morris EA. Screening breast MR imaging in women with a history of lobular carcinoma in situ. Radiology. 2011;261:414-420.

30. Brem RF, Fishman M, Rapelyea JA. Detection of ductal carcinoma in situ with mammography, breast specific gamma imaging, and magnetic resonance imaging: a comparative study. Acad Radiol. 2007;14:945-950.

31. Brem RF, Floerke AC, Rapelyea JA, Teal C, Kelly T, Mathur V. Breast-specific gamma imaging as an adjunct imaging modality for the diagnosis of breast cancer. Radiology. 2008;247:651-657.

32. O'Connor MK, Li H, Rhodes DJ, Hruska CB, Clancy CB, Vetter RJ. Comparison of radiation exposure and associated radiation-induced cancer risks from mammography and molecular imaging of the breast. Med Phys. 2010;37:6187-6198.

33. Heller SL, Elias K, Gupta A, Greenwood HI, Mercado CL, Moy L. Outcome of high-risk lesions at MRI-guided 9-gauge vacuum- assisted breast biopsy. AJR. 2014;202:237-245.

34. Lourenco AP, Khalil H, Sanford M, Donegan L. High-risk lesions at MRI-guided breast biopsy: frequency and rate of underestimation. AJR. 2014;203:682-686. 